\title{
Research on Electromagnetic Induction Signal Transmission Technology of Drilling Coal Sample Collection System
}

\author{
Xiaoliang Zheng ${ }^{1,3,{ }^{*}}$, Feng $\mathrm{Li}^{1}$, Liang Yuan $^{2}$, Sheng Xue ${ }^{3}$ and Zegong $\mathrm{Liu}^{3}$ \\ ${ }^{1}$ School of Electric and Information Engineering Anhui University of Science and Technology, China \\ ${ }^{2}$ Coal Mining National Engineering Technology Research Institute, China \\ ${ }^{3}$ School of Mining and Safety Engineering Anhui University of Science and Technology, China \\ *Corresponding author
}

\begin{abstract}
In view of the existing problems in the coal sample collection method of the coal seam gas content, a sampling method of fast sealing and sampling in coal seam drilling is proposed and the method's control signal can achieve the non-contact transmission by electromagnetic induction principle. The equivalent model of the electromagnetic induction transmission circuit is given, and the related laboratory experiments are carried out to verify the feasibility of the non-contact signal transmission. A series of conclusions are obtained: the sum of the voltages on the ideal state of all couplers is constant, and the voltage on each coupler is equal; the induced voltage is more stable when the transmission frequency is $240 \sim 310 \mathrm{kHz}$; increasing the number of coils' turns will improve the transmission efficiency, but it also increase the copper loss, so the most appropriate turns are around 40T; enlarging the coil gap will reduce the transmission efficiency, so the coil's gap should be reduced.
\end{abstract}

Keywords-coal mine safety; method based gas content; coal sample collection; electromagnetic induction; signal transmission

\section{INTRODUCTION}

Direct determination of gas content in underground coal seam is widely used in prediction of coal and gas outburst danger zone, evaluation of CBM resource, effect test of regional outburst measures, etc., with the advantages of high measuring speed and flexible measuring point. Deep hole coal sample collection device and technology are the basis of direct determination of gas content, and domestic outburst coal seam is generally relatively soft, so a complete coal core almost can not be got from it. Through the tireless efforts of researchers, a variety of coal sample collection device are developed for the domestic outburst seams' characteristics: core pipe, pressure air injection sampling device, reverse circulation positive pressure fast sampling device, access drill cuttings in front of drill hole,etc. [1]. In the direct determination method of gas content, the gas loss quantity is the important factor that affects the accuracy of gas content measurement [2]. The time length of the coal seam from exposing to be closed in the coal sampling can determine the amount of gas loss quantity. The exposure time of different sampling devices is different, and the shorter the time, the less the amount of gas will loss. To reduce the error of gas content determination, it is necessary to have a sampling system possessed of an automatic collection device that can automatically complete sampling in the deep hole, sealing and preserving. The drill pipes of coal sampling system are connected through the screw thread in together, it has been in rotation while drilling, and coal bed also has a certain shielding effect, so it is hard to transfer the control signal by commonly wired or wireless communication mode for the automatic sampling device in drilling depths. Electromagnetic induction signal transmission technology can meet the need of non-contact signal transmission, and the United States Intelliserv's intelligent drill pipe telemetry system has currently put into commercial application [3]. In order to achieve automatic control of coal sampling system, the electromagnetic induction transmission structure of Intelliserv can be used for referring to make further experimental study about electromagnetic induction signal transmission technology.

\section{ELECTROMAGNETIC INDUCTION SIGNAL TRANSMISSION TECHNOLOGY}

\section{A. Signal Coupling Principle}

Non-contact wireless transmission uses the principle of electromagnetic induction to achieve the transmission of signal. As the time-varying current through the conductor circuit, a varying electromagnetic field will be generated around the conductor; and if there is another circuit close to the conductor at this time, according to the principle of electromagnetic induction, the latter will be induced electromotive force [4].The coal sampling system makes full use of the principle and embed the ferrite ring groove which is wound with the induction coils at the end of each drill pipe's joint, so that when the excitation signal is applied to the previous stage, the latter stage of the coil will generate a response by the induction. The ferrite at both ends of the drill pipe are close, but not in direct contact, and that is the way which the signal passes through the gap.

\section{B. The Coupler's Equivalent Model}

The coal sampling system's channel is composed of inductive couplers and relay sections; and the coupler, as a very important transmission unit in the channel, is a key object on design and research. The principle of the coupler is similar to that of the transformer, and the operating frequency of the coupler is much higher than that of the ordinary transformer, so the model of the high frequency transformer can be used for reference. Then it is required to take into account the eddy 
current loss of the magnetic core, magnetic hysteresis loss, stray capacitance of the coil, distributed capacitance, etc. [5,6]. The difference between the coupler and the high frequency transformer is that: the channel of the coal sample collection system is required to realize the bidirectional signal transmission, so the turns of coils on both sides of the coupler must be consistent. The equivalent model of the coupler is shown in Figure I.

In Figure $I, C_{1}$ and $C_{2}$ are the equivalent capacitance of the induction coil; $\mathrm{R}_{1}$ and $\mathrm{R}_{2}$ are the equivalent $\mathrm{DC}$ loss of the induction coil; $\mathrm{R}_{\mathrm{m}}$ is the hysteresis loss and the eddy current loss of magnetic cores under high frequency condition; $\mathrm{C}_{\mathrm{p}}$ is the stray capacitance between the two coils; $\mathrm{L}_{1}$ is the magnetizing inductance of the primary coil. When the coupler's gap is very small, most of the flux of the primary coil will pass to the secondary coil except a small part will leak out, then the equivalent leakage inductance $\mathrm{L}_{11}$ and $\mathrm{L}_{12}$ are formed. As the leakage flux is equal to the total flux minus the flux surrounded by the secondary coil, the leakage inductance ought to be connected in series with $\mathrm{L}$.

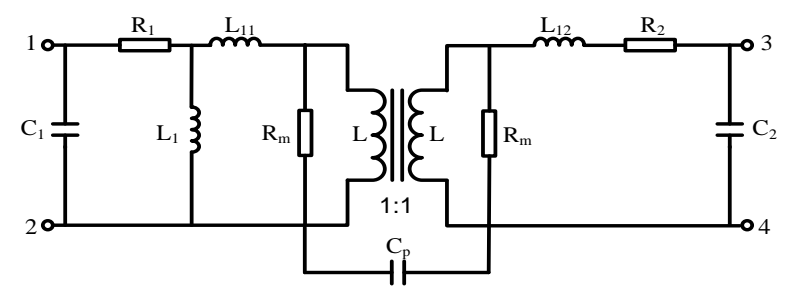

FIGURE I. EQUIVALENT MODEL OF THE COUPLER

\section{COAl SAmple COllection System StRuCture}

The structure of the coal sample collection system is shown in Figure II, and the terminal controller that is a terminal control module with a MCU as its core provides the operator with control interface, which is convenient to control the operating state of the coal sample collection system. The terminal controller can not only send the control signal to the collecting bin, but it can also receive feedback of the collecting bin's operating state and display it to the operator. The electromagnetic induction channel is composed of a plurality of drill pipes, each of which is embedded with an induction coil, and communication between the signal and the controller is connected by a modulation / demodulation circuit. The electromagnetic transmission channel may be composed of dozens or even hundreds of drill pipes, so the signal amplitude will attenuate in transmission process. Thus, it is necessary to add an relay section after a number of drill pipes to realize enhancement of the signal amplitude and bidirectional transmission of the signal.

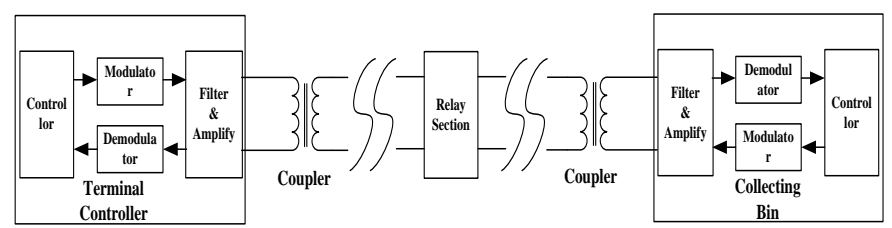

FIGURE II. STRUCTURE OF THE COAL SAMPLE COLLECTION SYSTEM
The signal transmission process is as follows: the dual requency sinusoidal carrier signal is obtained after the control signal sent out by the terminal controller is modulated, and the excitation current will be generated during the transmission process of the carrier signal. Magnetizing current make the primary coil of the drill pipe's joint generate an alternating electromagnetic field that could through the secondary coil, and the electromotive force will be induced by electromagnetic induction in the secondary coil. The induced electromotive force forms a new excitation source in the secondary coil, and the signal is transmitted to the next level in the same way. When collecting bin receives control signals after demodulation and began to execute instructions. After the action completed, the feedback signal is transmitted back to the terminal controller through the channel, and the staff can monitor the operating status of the collecting bin in real time through the display on the terminal controller.

\section{ELECTROMAGNETIC INDUCTION CHANNEL EXPERIMENT AND ANALYSIS}

\section{A. Transmission Characteristics}

In order to further explore the equivalent model of the electromagnetic induction channel, the transmission characteristics of the electromagnetic induction channel are analyzed by related experiments, and then it will be assumed and verified by the equivalent model of the coupler. The modulator selects XR2206 and the demodulator selects XR2211.The self-made couplers are connected to the XR2211 in turns. T1, T2, T3, T4, T5 represent the couplers and U1, U2, $\mathrm{U} 3, \mathrm{U} 4, \mathrm{U} 5$ are the induced voltage amplitude of the couplers. Five groups of data are obtained in the experiment and the number of coupler is different in each group. The first group only has T1; the second group has $\mathrm{T} 1$ and $\mathrm{T} 2$; the third group has T1, T2 and T3; the fourth group has T1,T2,T3 and T4; the fifth group has T1,T2,T3,T4 and T5. The amplitude of each group is shown in table 1 .

Such a law can be got from Table I:

$$
\sum_{\mathrm{i}=1}^{\mathrm{n}} \mathrm{U}_{\mathrm{i}} \approx 1.68 \mathrm{~V} \quad(\mathrm{n}=1,2,3,4,5) .
$$

TABLE I. COUPLERS' AMPLITUDE

\begin{tabular}{|c|c|c|c|c|c|}
\hline \multirow{2}{*}{$\begin{array}{c}\text { Group } \\
\mathrm{s}\end{array}$} & \multicolumn{5}{|c|}{ Amplitude } \\
\hline & U1 & U2 & U3 & U4 & U5 \\
\hline 1 & $1.68[\mathrm{~V}]$ & & & & \\
\hline 2 & $0.88[\mathrm{~V}]$ & $0.84[\mathrm{~V}]$ & & & \\
\hline 3 & $0.60[\mathrm{~V}]$ & $0.56[\mathrm{~V}]$ & $0.52[\mathrm{~V}]$ & & \\
\hline 4 & $0.48[\mathrm{~V}]$ & $0.44[\mathrm{~V}]$ & $0.40[\mathrm{~V}]$ & $0.36[\mathrm{~V}]$ & \\
\hline 5 & $0.40[\mathrm{~V}]$ & $0.36[\mathrm{~V}]$ & $0.32[\mathrm{~V}]$ & $0.32[\mathrm{~V}]$ & $0.28[\mathrm{~V}$ \\
\hline
\end{tabular}
electromagnetic induction channel can be assumed: with the increase of couplers' number, the sum of the voltages on all couplers is constant, and the voltage on each coupler is equal.

Further analysis of the equivalent model of the coupler: in the experiment, the operating frequency is lower than $1 \mathrm{MHz}$, which belongs to the low frequency circuit, then $C_{1}, C_{2}$ and $C_{p}$ that have no effect on the circuit can be ignore; due to the core 
loss is very small at low frequency, it can be ignored, so $\mathrm{R}_{\mathrm{m}}$ can be regarded as infinite; when the leakage inductance $L_{11}$ and $\mathrm{L}_{12}$ are much smaller than $\mathrm{L}$, they can be ignored; $\mathrm{R}_{1}$ and $\mathrm{R}_{2}$ are much less than $R_{S}$ in the real measurement, so they can be ignored. Thus, the practical equivalent circuit of the coupler in the low frequency circuit is shown in Figure III, and mesh equations of the current can be listed according to Kirchhoff's law,

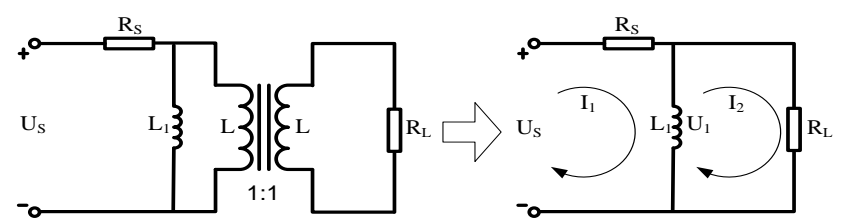

FIGURE III. PRACTICAL EQUIVALENT CIRCUIT OF THE COUPLER

$$
\begin{gathered}
\left(R_{S}+j \omega L_{1}\right) \dot{I}_{2}-j \omega L_{1} \dot{I}_{2}=\dot{U}_{S}, \\
\dot{I}_{1} j \omega L_{1}=\dot{I}_{2}\left(R_{L}+j \omega L_{1}\right) .
\end{gathered}
$$

Therefore,

$$
\frac{\dot{I}_{2}}{\dot{U}_{S}}=\frac{j \omega L_{1}}{\left(R_{S}+j \omega L_{1}\right)\left(R_{L}+j \omega L_{1}\right)-\left(j \omega L_{1}\right)^{2}}=\frac{j \omega L_{1}}{R_{L} R_{S}+\left(R_{L}+R_{S}\right) j \omega L_{1}}
$$

$$
\frac{\dot{U}_{1}}{\dot{U}_{S}}=\frac{j \omega L_{1} R_{L}}{R_{L} R_{S}+\left(R_{L}+R_{S}\right) j \omega L_{1}}=\frac{j \omega L_{1}}{R_{S}+j \omega L_{1}+\frac{R_{S} j \omega L_{1}}{R_{L}}}
$$

$R_{L}$ is the input resistance of XR2211, so $R_{L}$ is much larger than $R_{S}$, and $L_{1}$ is quite small, then $j \omega L_{1}$ is far less than $R_{S}+j \omega L_{1}$. Therefore,

$$
\dot{U}_{1}=\frac{j \omega L_{1}}{R_{S}} \dot{U}_{S}
$$

When $\mathrm{N}$ couplers are connected together, the transmission channel equivalent circuit is shown in Figure IV, and a conclusion can be obtained: the voltage of each coupler is equal to the voltage of the load, and $\mathrm{L}_{1}=\mathrm{L}_{2}=\mathrm{L}_{3}=\ldots=\mathrm{L}_{\mathrm{n}}$, then

$$
\dot{U}_{n}=\frac{j \omega L_{1}}{n R_{S}} \dot{U}_{S}
$$

\section{B. Analysis on the Influencing}

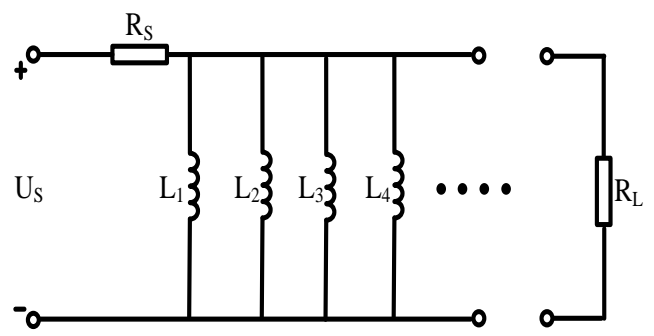

FIGURE IV.EQUIVALENT CIRCUIT OF TRANSMISSION CHANNEL

\section{Factors of Transmission Efficiency}

The transmission characteristics are obtained in the ideal condition; however, the transmission channel of electromagnetic induction used in practice will be affected by various factors, including the gap in the coupler, the turns of coils, the signal frequency and the choice of ferromagnetic materials, etc. [7,8]. In order to get a deeper understanding of the various problems that may be encountered in the practical use of the electromagnetic induction channel, the influence factors of transmission efficiency are researched by analyzing the relationship between electromagnetic signal transmission and coupler clearance, signal frequency, coil structure $[9,10]$.

Couplers adopt the $\mathrm{Mn}-\mathrm{Zn}$ ferrite as the core, its initial permeability is around 2300. The experiment uses the FSK modulation mode to transmit the signal, the controller will send square wave of $5 \mathrm{~V}$. The frequency $\mathrm{f}_{1}$ represents " 1 " code and the frequency $\mathrm{f}_{2}$ represents " 0 " code. Because the modulation and demodulation of FSK signal only care about the quality of waveform and amplitude, the transmission efficiency can be defined as: $\eta=v_{\mathrm{o}} / \mathrm{v}_{\mathrm{i}}, \mathrm{v}_{\mathrm{o}}$ is the secondary side induced voltage, $\mathrm{v}_{\mathrm{i}}$ is the primary side induced voltage.

1) Transmission Frequency: Couplers also act as a band-pass filter when it transmits signals, the difference of the coupler parasitic parameters also led its frequency-response characteristics to be different. Amplitude of the induced voltage on the coupler is an important symbol to test the efficiency of signal transmission. To change the XR2206's seventh pin and eighth pin of the adjustable resistance can make the waveform frequency vary in $50 \mathrm{kHz}$ to $350 \mathrm{kHz}$. The optimal transmission frequency can be found by using the oscilloscope to observe the condition of the secondary side induced voltage under different transmission frequencies. Figure $\mathrm{V}$ is the relationship between the voltage and the operating frequency. It can be seen in the Figure VI: the secondary side induced voltage is relatively stable when the operating frequency is from $240 \mathrm{kHz}$ to $310 \mathrm{kHz}$. 


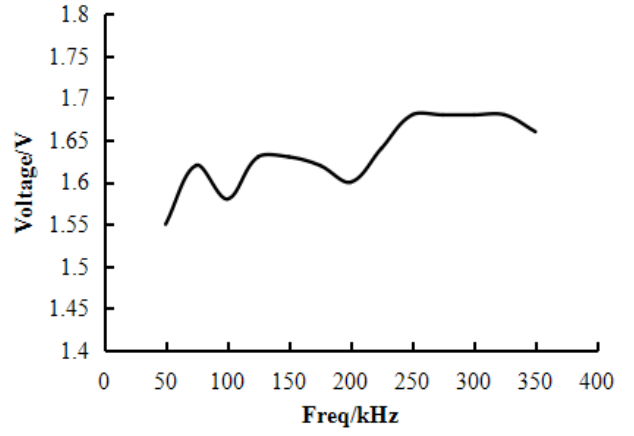

FIGURE V. CURVE OF THE INDUCED VOLTAGE AND THE OPERATING FREQUENCY

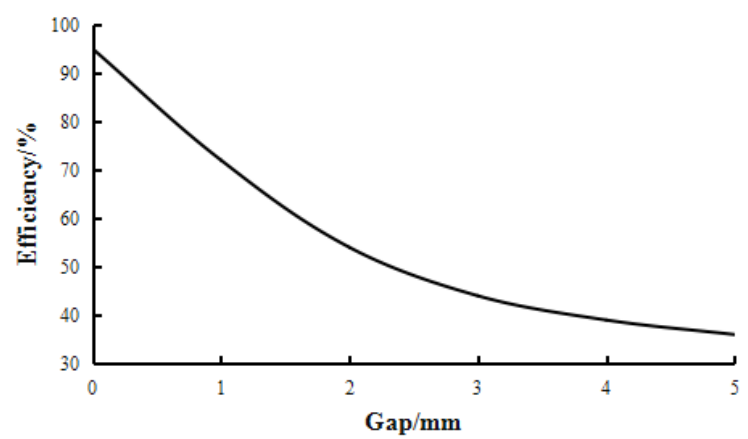

FIGURE VI.CURVE OF TRANSMISSION EFFICIENCY AND THE COUPLER'S GAP

2) The Coupler's Gap: Taking into account the presence of the certain gap between the drill pipe joints, and increasing the gap will inevitably reduce the mutual inductance coefficient of the coil, so it is important to control the size of the gap which is a direct impact on transmission efficiency of the electromagnetic induction channel. Figure VI is the relationship between the transmission efficiency and the coupler's gap. It can be seen from the figure that the transmission efficiency of the coil decreases sharply with the increase of the gap in the coupler. When the gap is $4 \mathrm{~mm}$, the transmission efficiency is less than $40 \%$.

3) The Turns of Coils: The change of turns of the coil directly affects the mutual inductance of the coil, and Figure VII is the relationship between the transmission efficiency and the turns of coils. It can be seen from the figure that the transmission efficiency increases with the increase of the turns of coils, especially it is more obvious around of 40 turns; although the turns increase of coils can improve transmission efficiency, but taking into account the copper loss increase and the channel bandwidth reduction, the turn are finally selected in 40T.

4) Magnetic Core Size: In the experiment, two sizes of magnetic cores are selected, the external diameter are $50 \mathrm{~mm}$, $10 \mathrm{~mm}$ and the internal diameter are $30 \mathrm{~mm}, 8 \mathrm{~mm}$. From the experimental data, it can be known that the magnetic core size can be enlarged to improve the transmission efficiency of electromagnetic induction. But the size of the magnetic core is limited by the size of the drill pipe's thread joint.

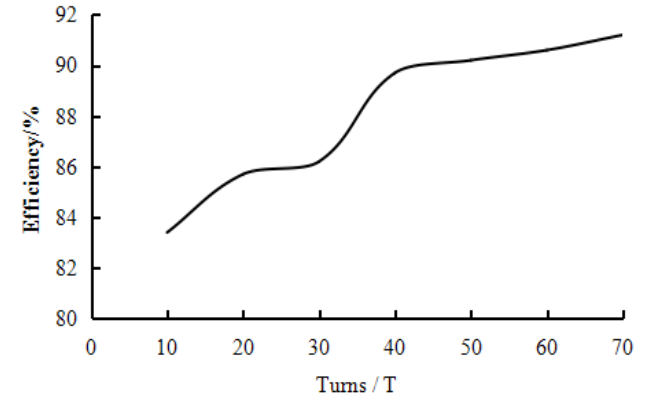

FIGURE VII.

\section{CURVE OF TRANSMISSION EFFICIENCY AND THE TURNS OF COILS}

\section{CONCLUSIONS}

It is feasible to realize the data transmission of coal sample collection system in underground coal seam by means of electromagnetic induction signal transmission, the sampling system can realize the fixed-point fast sampling, improve the accuracy of sampling position and shorten the time of coal sample exposure, so the accuracy of determination data of gas content in coal seam can be improved. The experimental results can be got that with the increase of drilling depths, that is the increase of couplers' number, the sum of the voltages on the ideal state of all couplers is constant, and the voltage on each coupler is equal. The induced voltage is reduced by the increase of the number of couplers, so it is necessary to add a relay section after a number of drill pipes to amplify the voltage.

In order to get a better signal transmission efficiency, the appropriate value of each parameter is determined by experiments: the induced voltage is more stable when the transmission frequency is $240 \sim 310 \mathrm{kHz}$; increasing the number of coils' turns will improve the transmission efficiency, but it also increase the copper loss, so the most appropriate turns are around 40T; enlarging the coupler's gap will reduce the transmission efficiency, so the coupler's gap should be reduced. The study provides a detailed experimental data for the further development of the system, and the new type of magnetic core material and the coil structure should be further studied to improve the transmission efficiency in the future.

\section{REFERENCES}

[1] Zhang Shutong. Key technology for gas content direct determination method in underground mine, Journal of Mining \& Safety Engineering.31(2014)328-332.

[2] Liang Wang, Long-biao Cheng, Yuan-ping Cheng, Shimin Liu, Pin-kun Guo, Kan Jin, Hai-na Jiang. A new method for accurate and rapid measurement of underground coal seam gas content. Journal of Natural Gas Science and Engineering.26(2015)1388-1398.

[3] Yue Chun-hong. Intelligent drill pipe-Will lead to a downhole signal transmission technology revolution, Journal of Petroleum Science and Technology Forum.4( 2008)34-36.

[4] Qiu Guan-yuan. Circuit, fifth ed, Higher Education Press,2006.

[5] Qin Hai-hong, Wand Hui-zhen, Yan Yang-guang. Analysis and Design of Contactless Loosely Coupled Inductive Power Transfer System, Journal of Power Supply Technologies and Applications. 17(2004) 257-262.

[6] Fu Wen-zhen, Zhang Bo, Qiu Dong-yuan, Wang Wei. Maximum Efficiency Analysis and Design of Self-resonance Coupling Coils for Wireless Power Transmission System, Journal of Proceedings of the CSEE.29(2009)22-26. 
[7] Wu Zhong-hua, Sun Hao-yu, Zhang Shi-ping, Zhu Zheng. Research on data transmission system of electromagnetic induction type, Journal of China Petroleum Machinery.37(2009)58-60.

[8] Xiong Chen-long, Sheng bing, Zhao Ning. Simulation Research on Energy Transmission Efficiency of Wireless Charging Technology Based on the Electromagnetic Induction, Journal of Electron Devices.37(2014)132-133.

[9] He Zhi-yuan, Kang Min. Analysis of a Rectangular Magnetic Charging Coil by Finite Element Method, Journal of System Simulation.22(2010)2813-2816.

[10]Jiang Da-qing, Fu Zhi-hong, Hou Xing-zhi. Zhang Huai-qing. Research about 3D Forward Modeling of Transient Electromagnetic Method Based on Maxwell 3, Journal of Electrical Measurement \& Instrumentation.49(2012)29-32. 